\title{
A NEW PROOF AND GENERALIZATIONS OF THE CAUCHY-KOWALEWSKI THEOREM( $\left.{ }^{1}\right)$
}

\author{
BY \\ AVNER FRIEDMAN
}

Introduction. In 1842 Cauchy proved an existence theorem for analytic nonlinear differential equations of the second order, which was extended in 1875 by S. Kowalewski to general analytic nonlinear systems of differential equations and became known as the Cauchy-Kowalewski theorem. In his proof $[1 ; 6]$ Cauchy uses the method of majorants (which he calls "Calcul de limites"). This method consists of (a) inserting an analytic expansion for the solution into the differential equation and thus obtaining recursive formulas for the coefficients of the expansion, and (b) estimating these coefficients and thus proving that the formal solution is a genuine one. The essence of the method lies in the fact that a direct estimation can be avoided. Indeed, Cauchy constructs a simple "majorant problem" which can be solved explicitly and its solution establishes the desired estimates.

In $1874 \mathrm{~S}$. Kowalewski, apparently unaware of the work of Cauchy, extended (in her thesis) his results to general nonlinear analytic systems. Her work, which appeared in 1875 [18] contains the result of Darboux [7] (the same year) who has also extended the work of Cauchy. The proof as it is known today is a simplification of the proof of Kowalewski and it is due to Goursat $[15]$ (see also [16; p. 360]). All the above proofs are based on the very same ideas of Cauchy, mentioned above. A method based on direct estimates of the coefficients was given by P. Lax [19]; see $\$ 3$.

Some work has been done in the direction of extending the C-K (CauchyKowalewski) theorem to nonanalytic equations. For the system

$$
\frac{\partial^{2} u}{\partial t^{2}}=\frac{\partial u}{\partial x}, u(x, 0)=\phi_{1}(x), \frac{\partial u(x, 0)}{\partial t}=\phi_{2}(x),
$$

it was proved by LeRoux [20] in 1895 and by Holmgren [17] in 1908 that if $\phi_{1}, \phi_{2}$ belong to class 2 of Gevrey (that is, if $\left|\partial^{n} \phi_{i} / \partial x^{n}\right| \leqq A_{0} A^{n}(2 n)$ ! for some constants $A_{0}, A$ and for all $n \geqq 0$ ) then there exists one and only one analytic (in $t$ ) solution. This result was extended in the last decade to more general equations. Salehov and Friedlender [10], Pucci [21], Friedlender [11] and others mentioned in $[10 ; 11]$ considered various special linear equations. In 1959 Friedlender [12] extended this also to a special class of nonlinear equations. The method he uses is based on the same scheme ((a), (b) above) of

Received by the editors May 9, 1960.

(1) Prepared under Contract Nonr 710(16) (NR 044 004) between the Office of Naval Research and the University of Minnesota. 
Cauchy, but he derives direct estimates on the derivatives. The estimates are not strong enough to solve the problem for general nonlinear equations.

In this paper we give a new proof of the C-K theorem (for general nonlinear systems), and in fact, we prove a more general theorem which allows certain degree of nonanalyticity in the system. In particular, our theorem contains as very special cases all the above mentioned results about nonanalytic equations. Our proof is based on the following simple observation: By differentiating appropriately the differential equation one obtains recursive formulas for the derivatives of the solution, provided it exists. Next, one may try to obtain successively a priori bounds on the derivatives of the solution. Finally, after these bounds have been derived, one can construct the solution as a power series in the distinguished variable with coefficients obtained from the recursive formulas (and for which, therefore, we have already a priori bounds).

In order to accomplish this program we must know how to estimate the derivatives of a function $f(u(x))$ nonlinear in $u$, when we are given bounds on the derivatives of $u$. Such a problem we have already encountered in our work [13] on the differentiability and analyticity of solutions of nonlinear elliptic systems. We use some essential ideas of that paper for the treatment of the present problem.

In Part I we prove a generalization of the $\mathrm{C}-\mathrm{K}$ theorem for one equation. For the sake of clarity we first prove it for a special nonlinear equation and then for the general equation. In Part II we prove a theorem which generalizes the C-K theorem for general (nonlinear) systems. We note that in our proof it is not necessary to reduce the given systems to a first-order system as is done in the classical proof. In Part III we generalize the results of Part I to the case of several distinguished variables. More precisely, we prove a theorem which contains, in particular, a solution of the Goursat problem for an analytic equation. A special result was derived in [12]. Finally, in Part IV we consider systems which contain as a very special case systems introduced by Duff [8] in his study of mixed problems.

\section{Part I. The case of one equation}

1. Notations and results. In Part I we shall consider the case of one equation and prove a theorem which contains as a special case the $\mathrm{C}-\mathrm{K}$ theorem for one equation. For the sake of clarity we first state a typical special case (Theorem 1) and then state the general result (Theorem 2). Theorem 1 is proved in $\$ \S 2-4$ and Theorem 2 is proved in $\$ 5$. We need a few definitions and notations.

Let $x=\left(x_{1}, \cdots, x_{N}\right), v=\left(v_{1}, \cdots, v_{s}\right)$ where $x_{i}, v_{j}$ are real variables. By a region we mean a domain plus a part of its boundary. We define $D_{t}=\partial / \partial t$ for $t$ real, $D_{x}=$ any partial derivative with respect to one of the $x_{i}$; similarly for $D_{*}$. A function $g(x, t, v)$ is said to belong to class $\left(\delta_{1}, \delta_{2}, \delta_{3}\right)$ in a region $D$ 
( $\delta_{i}$ non-negative), if $g(x, t, v)$ is indefinitely differentiable in $D$, and if there exist constants $H_{0}, H$ such that for any $(x, t, v)$ in $D$,

$$
\left|D_{x}^{i} D_{t}^{j} D_{v g}^{k}(x, t, v)\right| \leqq H_{0} H^{i+j+k}\left(\delta_{1} i\right) !\left(\delta_{2} j\right) !\left(\delta_{3} k\right) !(i, j, k=0,1,2, \cdots)
$$

where, by definition, $\left(\delta_{1} i\right) !=\Gamma\left(\delta_{1} i+1\right)$, etc. For later use we also define $a !=0$ for any $a \leqq 0$. Note that if one of the $\delta_{k}$ is equal to 1 , then $g$ is analytic in the corresponding variable. Note also that if we replace the inequalities (1.1) by the inequalities

$$
\left|D_{x}^{i} D_{t}^{j} D_{v}^{k} g(x, t, v)\right| \leqq H_{0} H_{1}^{i} H_{2}^{j} H_{3}^{k}\left(\delta_{1} i-\alpha_{1}\right) !\left(\delta_{2} j-\alpha_{2}\right) !\left(\delta_{3} k-\alpha_{3}\right) !
$$

for any constants $\alpha_{1}, \alpha_{2}, \alpha_{3}$ depending on $g$, then we obtain the same class $\left(\delta_{1}, \delta_{2}, \delta_{3}\right)$. Also note that if we replace (1.1) by the apparently weaker inequalities

$$
\left|D_{x}^{i} D_{t}^{j} D_{v}^{k} g(x, t, v)\right| \leqq H_{0} H^{i+j+k}\left(\delta_{1} i+\delta_{2} j+\delta_{3} k\right) !
$$

we again get the same class $\left(\delta_{1}, \delta_{2}, \delta_{3}\right)$. The proof is immediate in view of the inequality $\left(\gamma_{i} \geqq 0\right)$

$$
\left(\gamma_{1} m+\gamma_{2} n\right) ! \leqq M_{0} M^{m+n}\left(\gamma_{1} m\right) !\left(\gamma_{2} n\right) !
$$

for some $M_{0}, M$ independent of $m, n$.

If $g=g(x, t)$ is of class $\left(\delta_{1}, \delta_{2}, \delta_{3}\right)$, then we say that $g$ is of class $\left(\delta_{1}, \delta_{2}\right)$.

We now consider the differential equation

$$
D_{t}^{p} u=f\left(x, t, D_{x}^{q} D_{t}^{r} u\right), \quad(q+r \leqq p, r<p),
$$

where $x$ varies in a bounded region $B$ and $|t| \leqq t_{0} \cdot\left(t_{0}>0\right)$. We take, for the initial conditions,

$$
D_{t}^{k} u(x, 0)=0, \quad 0 \leqq k \leqq p-1 .
$$

In order to consider the system (1.4), (1.5), $f(x, t, v)$ ( $v$ a real variable) must be defined for $x \in B,|t| \leqq t_{0},|v| \leqq v_{0}$ for some $v_{0}>0$. Note that the condition $q+r \leqq p$ appears in the classical $\mathrm{C}-\mathrm{K}$ theorem; thus it follows that $(p-r) / q \geqq 1$.

TheOREM 1. Let $f(x, t, v)$ be of class $(\delta, 1,1)$ in the region defined by $x \in B$, $|t| \leqq t_{0},|v| \leqq v_{0}$, where $1 \leqq \delta \leqq(p-r) / q$. Then there exists one and only one solution $u(x, t)$ of (1.4), (1.5) which is analytic in $t$ near $t=0$. Furthermore, $u(x, t)$ is of class $(\delta, 1)$ for $x \in B,|t| \leqq t_{1}$ for some $t_{1}>0$ sufficiently small.

Note that the condition $\delta \leqq(p-r) / q$ is sharp, since it is sharp already for the special equation $D_{t} u=D_{x}^{2} u[10 ; 17 ; 20]$.

We next state the general theorem, which contains Theorem 1 . It considers the differential equation 


$$
D_{t}^{p} u=f\left(x, t, u, \cdots, D_{x}^{q_{i}} D_{t}^{r_{i}} u, \cdots\right), \quad\left(q_{i}+r_{i} \leqq p, r_{i}<p\right) .
$$

THEOREM 2. Let $f(x, t, v)$ (where $v=\left(v_{1}, \cdots, v_{s}\right)$ ) be of class $(\delta, 1,1)$ in the region defined by $x \in B,|t| \leqq t_{0}, \sum v_{i}^{2} \leqq v_{0}^{2}$, where $1 \leqq \delta \leqq \min _{i}\left(p-r_{i}\right) / q_{i}$. Then there exists one and only one solution $u(x, t)$ of $(1.6),(1.5)$ which is analytic in $t$ near $t=0$. Furthermore, $u(x, t)$ is of class $(\delta, 1)$ for $x \in B,|t| \leqq t_{1}$ for some $t_{1}>0$ sufficiently small.

The case of nonhomogeneous initial conditions

$$
D_{t}^{k} u(x, 0)=\phi_{k}(x) \quad(0 \leqq k \leqq p-1),
$$

with the $\phi_{k}$ being of class $\delta$, easily follows from Theorem 2 by substituting

$$
v(x, t)=u(x, t)-\sum_{k=0}^{p-1} \frac{t^{k}}{k !} \phi_{k}(x) .
$$

Here we use the fact that an analytic function of a function of class $\delta$ is of class $\delta$. (This follows, for instance, by the considerations of [11].) It thus follows that Theorem 2 remains true also for the system (1.6), (1.7), provided the $\phi_{k}(x)$ are of class $\delta$.

Note that in the special choice $\delta=1$ we obtain the C-K theorem for one differential equation.

From the proof of Theorems 1, 2 it will become clear that the following generalization is true:

If $f\left(x_{1}, \cdots, x_{n}, t, v\right)$ is of class $\delta_{j}$ in $x_{j}(1 \leqq j \leqq N)$ and of class $(1,1)$ in $(t, v)$ and if $1 \leqq \delta_{j} \leqq \min _{i}\left(p-r_{i}\right) / q_{v}$, then the solution $u(x, t)$ is of class $\delta_{j}$ in each $x_{j}$ and of class 1 in $t$.

This generalization can also be appropriately extended for the case of the nonhomogeneous initial conditions (1.7), for the case of differential systems discussed in Part II, for the Goursat problem discussed in Part III, and for the mixed problems of Part IV.

In $\S 2$ we reduce the proof of Theorem 1 to the proof of deriving, by induction, a priori estimates on the derivatives of the solution. To establish these estimates, we need a lemma which is proved in $\$ 3$. The proof of Theorem 1 is completed in $\S 4$. In $\S 5$ we modify the proof of Theorem 1 and thus derive a proof of Theorem 2.

2. Reduction to recursive inequalities. Suppose there exists an analytic solution $u(x, t)=\sum u_{n}(x) t^{n}$. Then, by (1.5), $u_{k}(x) \equiv 0$ for $0 \leqq k \leqq p-1$, and by (1.4) the successive $t$-derivatives of $u(x, t)$ at $t=0$ are determined recursively in a unique manner. This proves the uniqueness of the $u_{n}(x)$ and, hence, of the solution $u(x, t)$. To prove existence, we shall establish by induction that

$$
\left|D_{x}^{m} D_{t}^{k} u(x, 0)\right| \leqq H_{0} H^{m+2 k-q-2 r-1}(\delta m+k-\delta q-r-4) !
$$

for all $k \geqq p, m \geqq 0$ (which implies that $m+2 k \geqq q+2 r+1$ ). 
Once (2.1) is established, the proof of Theorem 1 can be completed as follows. We construct a function

$$
\tilde{u}(x, t)=\sum_{k=p}^{\infty} u_{k}(x) t^{k} \text { where } k ! u_{k}(x)=D_{t}^{k} u(x, 0) .
$$

By (2.1), $\tilde{u}(x, t)$ is analytic in $t$. Hence, in order to prove that it satisfies (1.4), we have only to prove that if we differentiate both sides of $D_{i}^{p} \tilde{u}(x, t)$ $=f\left(x, t, D_{x}^{q} D_{z}^{r} \tilde{u}(x, t)\right) n$ times with respect to $t$ (for any $n \geqq 0$ ) and then substitute $t=0$, we obtain equality. Now, the desired equality is exactly the equation by which we have recursively defined $D_{t}^{n+p} u(x, 0)$, and since $D_{t}^{k} u(x, 0)$ $=\left.D_{t}^{k} \tilde{u}(x, t)\right|_{t=0}$, our proof is completed. Hence, $\tilde{u}(x, t)$ is a solution of (1.4). It thus remains to prove that $\tilde{u}(x, t)$ is of class $(\delta, 1)$.

Now by (2.2), (2.1) we conclude that

$$
k !\left|D_{x}^{m} u_{k}(x)\right|=\left|D_{x}^{m} D_{t}^{k} u(x, 0)\right| \leqq C_{1} H_{1}^{m+k}(\delta m+k) !
$$

for some constants $C_{1}, H_{1}$. Hence

$$
\begin{aligned}
\left|D_{x}^{m} D_{t}^{n} \tilde{u}(x, t)\right| & \leqq \sum_{k=n}^{\infty} k(k-1) \cdots(k-n+1) C_{1} H_{1}^{m+k} \frac{(\delta m+k) !}{k !}|t|^{k-n} \\
& \leqq C_{2} H_{2}^{m+n}(\delta m+n) !
\end{aligned}
$$

for some constants $C_{2}, H_{2}$. This completes the proof of Theorem 1 . It thus remains to prove (2.1).

We remark if we replace (2.1) by the simpler inequalities

$$
\left|D_{x}^{m} D_{t}^{n} u(x, 0)\right| \leqq H_{0} H^{m+k}(\delta m+k) !
$$

then our method, given below, fails. The reason will be made clear at the end of $\S 3$.

In order to prove (2.1), we first choose $H_{0} \geqq 1$ such that

$$
\left|D_{x}^{m} D_{t}^{k} u(x, 0)\right| \leqq H_{0} \text { if } m+2 k \leqq q+2 r+1 .
$$

(Actually, if $m+2 k<q+2 r+1$ then, necessarily, $k<p$ and, hence, the left side of (2.4) vanishes.) The constant $H$ will be determined in the inductive passage from $k$ to $k+1$. We assume that $(2.1)$ holds for all $k<n+p(n \geqq 0)$ and all $m \geqq 0$, and we shall prove it for $k=n+p$ and all $m \geqq 0$, that is (in view of (2.4)) we shall prove that

$$
\left|D_{x}^{m} D_{t}^{n+p} u(x, 0)\right| \leqq H_{0} H^{m+2 n+2 p-q-2 r-1}(\delta m+n+p-\delta q-r-4) !
$$

for all $m \geqq 0$ for which $m+2 n+2 p \geqq q+2 r+2$. We shall need a lemma which we prove in the following paragraph. 
3. An elementary lemma. Let $W(x, t)$ be a function satisfying

$$
\left|D_{x}^{m} D_{t}^{k} W(0,0)\right| \leqq U_{m k} \text { for } 0 \leqq k \leqq n, 0 \leqq m<\infty .
$$

Then we write

$$
W \stackrel{n}{\ll} U
$$

where

$$
U(x, t)=\sum_{m=0}^{\infty} \sum_{k=0}^{\infty} U_{m k} \frac{\left(x_{1}+\cdots+x_{N}\right)^{m}}{m !} \frac{t^{k}}{k !}
$$

where the definition of $U_{m k}$ for $k>n$ is immaterial; for simplicity we may sometimes take $U_{m k}=0$ for $k>n$. We say that $U(x, t)$ majorizes $W(x, t)$ at $(0,0)$ up to degree $n$. Note that the power series of $U$ is only a formal series; it need not be convergent. We shall be concerned with the following question: Given a $U$ which majorizes $W$, find $U_{j}$ which majorizes $W^{i}$, for each $j \geqq 2$. More specifically, we assume that

$$
\left|D_{x}^{m} D_{t}^{k} W(0,0)\right| \leqq\left\{\begin{array}{lr}
M_{0} M^{m+2 k-1}(\delta m+k-4) ! & \text { if } 1 \leqq k \leqq n, \\
0 & \text { if } k=0,
\end{array}\right.
$$

where $M \geqq 1$. We shall prove:

Lemma 1. Assume that (3.1) holds for all $m \geqq 0$. Then there exists a constant $K$, depending only on $\delta$, such that, if $j \geqq 1$,

$$
W^{i} \stackrel{n}{K}_{m=0}^{\infty} \sum_{k=1}^{\infty}\left(\frac{K M_{0}}{M}\right)^{i-1} M_{0} M^{m+2 k-1}(\delta m+k-4) ! \frac{\left(x_{1}+\cdots+x_{N}\right)^{m}}{m !} \frac{t^{k}}{k !} .
$$

Proof. For the sake of simplicity we agree to write $D_{x}^{m}=D_{x}^{s}$ whenever $m=s$. Since our estimates do not depend on the particular variables with respect to which the partial differentiation is performed, this agreement is justified. We then can use the Leibnitz law:

$$
D_{x}^{m} W^{j}=D_{x}^{m}\left(W^{j-1} W\right)=\sum_{\lambda=0}^{m}\left(\begin{array}{c}
m \\
\lambda
\end{array}\right) D_{x}^{\lambda} W^{j-1} D_{x}^{m-\lambda} W .
$$

From now on to the end of the proof, $C$ will be used to denote any positive constant depending only on $\delta$.

We proceed to prove (3.2) for $j=2$. Using (3.3), (3.1) we get

$$
\begin{array}{r}
\left|D_{2}^{m} D_{t}^{n} W^{2}\right|_{(x, t)-(0,0)} \leqq \sum_{\mu=0}^{m}\left(\begin{array}{l}
m \\
\mu
\end{array}\right) \sum_{\nu=1}^{n-1}\left(\begin{array}{l}
n \\
\nu
\end{array}\right)\left|D_{x}^{\mu} D_{t}^{\nu} W\right|\left|D_{x}^{m-\mu} D_{t}^{n-\nu} W\right| \\
\leqq \sum_{\mu=0}^{m}\left(\begin{array}{l}
m \\
\mu
\end{array}\right) \sum_{\nu=1}^{n-1}\left(\begin{array}{l}
n \\
\nu
\end{array}\right) M_{0} M^{\mu+2 \nu-1}(\delta \mu+\nu-4) ! M_{0} M^{m-\mu+2 n-2 \nu-1} \\
\cdot(\delta m-\delta \mu+n-\nu-4) ! .
\end{array}
$$


We proceed to estimate

$$
I \equiv \sum_{\mu=0}^{m} \sum_{\nu=1}^{n-1}\left(\begin{array}{l}
m \\
\mu
\end{array}\right)\left(\begin{array}{l}
n \\
\nu
\end{array}\right)(\delta \mu+\nu-4) !(\delta m-\delta \mu+n-\nu-4) !
$$

and, in fact, prove that

$$
I \leqq C(\delta m+n-4) !
$$

Since this inequality holds for each separate term in the sum of $I$, it is enough to consider

$$
I^{\prime}=\sum_{4 \leqq \delta \mu+\nu \leqq \delta m+n-4}\left(\begin{array}{l}
m \\
\mu
\end{array}\right)\left(\begin{array}{l}
n \\
\nu
\end{array}\right)(\delta \mu+\nu-4) !(\delta m-\delta \mu+n-\nu-4) ! .
$$

There are two possibilities: either $\delta \mu+\nu \geqq(\delta m+n) / 2$ or $\delta \mu+\nu<(\delta m+n) / 2$. It is enough to consider the partial sum corresponding to the first possibility (we call it $I^{\prime \prime}$ ) since the remaining sum can be treated similarly (by replacing $\mu, \nu$ by $m-\mu, n-\nu)$. We have

$$
\begin{aligned}
I^{\prime \prime} & \leqq C \underset{\mu, \nu}{\text { l.u.b. }}\left(\begin{array}{c}
m \\
\mu
\end{array}\right)\left(\begin{array}{l}
n \\
\nu
\end{array}\right)(\delta \mu+\nu-4) !(\delta m-\delta \mu+n-\nu) ! \sum_{i, j>0} \frac{1}{(i+j)^{4}} \\
& \leqq \frac{C}{(\delta m+n)^{4}} \operatorname{liu.b}_{\mu, \nu}\left(\begin{array}{l}
m \\
\mu
\end{array}\right)\left(\begin{array}{l}
n \\
\nu
\end{array}\right)(\delta \mu+\nu) !(\delta m-\delta \mu+n-\nu) ! \\
& \leqq C(\delta m+n-4) !
\end{aligned}
$$

where the last inequality can be verified directly. Indeed, for $\delta=1$ this reduces to

$$
\left(\begin{array}{c}
\mu+\nu \\
\mu
\end{array}\right)\left(\begin{array}{c}
m-\mu+n-\nu \\
m-\mu
\end{array}\right) \leqq\left(\begin{array}{c}
m+n \\
m
\end{array}\right)
$$

whereas if $\delta>1$ we also have to use the inequality, for $\mu>1 /(\delta-1)$, $m-\mu>1 /(\delta-1)$,

$$
\begin{aligned}
(\mu+1+\nu) \cdots([\delta \mu]+\nu) \frac{(\delta \mu+\nu) !}{([\delta \mu]+\nu) !}(m-\mu+n-\nu) & \cdots \\
\cdot([\delta m-\delta \mu]+n-\nu) & \frac{(\delta m-\delta \mu+n-\nu) !}{([\delta m-\delta \mu]+n-\nu) !} \\
\leqq & (m+1+n) \cdots([\delta m]+n) \frac{(\delta m+n) !}{([\delta m]+n) !}
\end{aligned}
$$

Substituting the estimate on $I$ into (3.4), we get

$$
\begin{aligned}
\left|D_{x}^{m} D_{t}^{n} W^{2}\right| & \leqq C M_{0}^{2} M^{m+2 n-2}(\delta m+n-4) ! \\
& \leqq\left(\frac{K M_{0}}{M}\right) M_{0} M^{m+2 n-1}(\delta m+n-4) !
\end{aligned}
$$


The proof for $j=2$ is thus completed.

The proof for $j=3$ follows exactly in the same manner, by using (3.3) with $j=3$, and applying (3.1) and (3.2) with $j=2$. The passage from $j$ to $j+1$, for any $j$, is also proved in the same manner. We therefore omit further details.

REMARK. The importance of Lemma 1 is that it shows that if we have a priori bounds on the derivatives of $W$, we then have also a priori bounds on the derivatives of $\sum_{j} A_{j} W^{i}$ provided $\left|A_{j}\right| \leqq$ const. $\left\langle\infty, M>K M_{0}\right.$, and that the latter bounds are of the same order of magnitude as the bounds corresponding to $W$. This is precisely the kind of estimates which are required in handling nonlinear problems (compare [13]). If we replace (3.1) by

$$
\left|D_{x}^{m} D_{i}^{k} W\right| \leqq M_{0} M^{m+2 k}(\delta m+k) ! \equiv U_{m k}
$$

then the coefficients of $U^{2}, U^{3}$, etc. will increase with the powers of $U$ and we shall not obtain useful majorants for $W^{2}, W^{3}$, etc.

In order to be able to use Lemma 1 we assumed (2.1) instead of (2.3). Thus, we assume slightly stronger bounds and prove slightly stronger bounds.

In [19] P. Lax proves the $\mathrm{C}-\mathrm{K}$ theorem for first order systems of ordinary differential equations and, as he mentions, the proof can be extended to first order partial differential equations. He first writes down a formal power series for the solution and then estimates the coefficients directly with the aid of the power series $f(x)=\sum x^{i} /(j+1)^{2}$ which has already been introduced by Gevrey [14]. The underlying idea in introducing the above function is similar to that which led us, in a different way, to introduce Lemma 1.

4. Completion of the proof of Theorem 1. Throughout this paragraph $C$ will be used to denote any constant independent of $H$. By assumption we have

$$
f(x, t, v)=\sum_{j=0}^{\infty} a_{j}(x, t) v^{j}
$$

where

$$
\left|D_{x}^{m} D_{t}^{n} a_{j}(x, t)\right| \leqq A_{0} A^{m+2 n+j}(\delta m+n-4) ! \quad(m, n=0,1,2, \cdots) .
$$

We now proceed to prove (2.5) for any $x$ in $B$. Without loss of generality we may take $x=0$, since the general case follows immediately by translation of $x$. By (1.4), (4.1), we have

$$
\begin{aligned}
& \left|D_{x}^{m} D_{t}^{n+p} u(0,0)\right|=\left|D_{x}^{m} D_{t}^{n} f\left(x, t, D_{x}^{q} D_{t}^{r} u(x, t)\right)\right|_{(x, t)-(0,0)} \\
& \leqq\left|D_{x}^{m} D_{t}^{n} a_{0}(0,0)\right|+\sum_{\mu=0}^{m}\left(\begin{array}{c}
m \\
\mu
\end{array}\right) \sum_{\nu=p-r}^{n}\left(\begin{array}{l}
n \\
\nu
\end{array}\right) \\
& \cdot \sum_{j \geq 1}\left|D_{x}^{m-\mu} D_{t}^{n-\nu} a_{j}(0,0)\right|\left|D_{x}^{\mu} D_{t}^{p}\left[D_{x}^{q} D_{t}^{r} u(0,0)\right]^{i}\right|
\end{aligned}
$$


Note that if $n<p-r$ the second term on the right side of (4.3) does not appear. It will be enough to consider the case $n \geqq p-r$.

Since $\nu+r<n+p$, we can apply Lemma 1 with

$$
W(x, t)=D_{x}^{q} D_{t}^{r} u(x, t)
$$

(Here we make use of the inductive assumption (2.1) with $k<n+p$.) We conclude that

$$
\left|D_{x}^{\mu} D_{t}^{\nu}\left[D_{x}^{q} D_{t}^{r} u\right]^{j}\right| \leqq\left(\frac{K H_{0}}{H}\right)^{j-1} H_{0} H^{\mu+2 v-1}(\delta \mu+\nu-4) !
$$

Substituting (4.4) into (4.3) and using (4.2), we get

$$
\begin{aligned}
& \left|D_{x}^{m} D_{t}^{n+p} u\right| \\
& \leqq \sum_{\mu=0}^{m}\left(\begin{array}{l}
m \\
\mu
\end{array}\right) \sum_{\nu=1}^{n}\left(\begin{array}{l}
n \\
\nu
\end{array}\right) \sum_{j} A_{0} A^{m-\mu+2 n-2 \nu+j}(\delta m-\delta \mu+n-\nu-4) ! \\
& \times\left(\frac{K H_{0}}{H}\right)^{j-1} H_{0} H^{\mu+2 v-1}(\delta \mu+\nu-4) !+A_{0} A^{m+2 n}(\delta m+n-4) ! .
\end{aligned}
$$

To estimate the sum we take $H>2 K H_{0} A, H>2 A$ and find the upper bound

$$
\begin{array}{r}
C H_{0} H^{m+2 n-1} \text { l.u.b. }\left(\begin{array}{l}
m \\
\mu, \nu
\end{array}\right)\left(\begin{array}{l}
n \\
\nu
\end{array}\right)(\delta m-\delta \mu+n-\nu-4) !(\delta \mu+\nu-4) ! \\
\quad \leqq C H_{0} H^{m+2 n-1}(\delta m+n-4) ! .
\end{array}
$$

Hence,

(4.6) $\left|D_{x}^{m} D_{t}^{n+p} u(0,0)\right| \leqq C H_{0} H^{m+2 n-1}(\delta m+n-4) ! \leqq H_{0} H^{m+2 n}(\delta m+n-4) !$

provided $H \geqq C$. To complete the proof of $(2.5)$, we only have to verify that

$$
\begin{gathered}
m+2 n \leqq m+2 n+2 p-q-2 r-1, \\
\delta m+n-4 \leqq \delta m+n+p-\delta q-r-4 .
\end{gathered}
$$

These inequalities reduce to

$$
2(p-r) \geqq q+1, \quad \delta q \leqq p-r .
$$

The first one follows by recalling that $p-r \geqq q, p-r \geqq 1$, whereas the second inequality follows by assumption (here is the only place where the restriction on the upper bound of $\delta \geqq 1$ is used).

REMARK. We could take the power of $H$ on the right side of (2.1) to be

$$
m+\gamma k-q-\gamma r-1
$$


for any $\gamma$ satisfying $\gamma(p-r)>q$ and then the above proof remains valid with slight modifications.

5. Proof of Theorem 2. Let $(p, q)$ be a pair $\left(p_{j}, q_{j}\right)$ which satisfies:

$$
\delta q+r \equiv \delta q_{j}+r_{j}=\max _{i}\left(\delta q_{i}+r_{i}\right) \text {. }
$$

Let $\left(q^{\prime}, r^{\prime}\right)$ be a pair $\left(p_{k}, q_{k}\right)$ which satisfies

$$
q^{\prime}+2 r^{\prime} \equiv q_{k}+2 r_{k}=\max _{i}\left(q_{i}+2 \dot{r}_{i}\right) \text {. }
$$

We now proceed as in the proof of Theorem 1, with some modifications. We replace $(2.1)$ by

$$
\left|D_{x}^{m} D_{t}^{k} u(x, 0)\right| \leqq H_{0} H^{m+2 k-q^{\prime}-2 r^{\prime}-1}(\delta m+k-\delta q-r-4) ! .
$$

Instead of (4.1) we have

$$
f(x, t, v)=\sum a_{j_{1}} \cdots j_{e}(x, t) v_{1}^{j_{1}} \cdots v_{\text {s }}^{j_{\text {s }}}
$$

where

$$
\left|D_{x}^{m} D_{t}^{n} a_{j_{1} \ldots j_{s}}(x, t)\right| \leqq A_{0} A^{m+2 n+j_{1}+\cdots+j_{8}}(\delta m+n-4) ! .
$$

In analogue with (4.3) we have (for $n \geqq p-r_{i}$, which is assumed for the sake of simplicity)

$$
\left|D_{x}^{m} D_{t}^{n+p} u(0,0)\right| \leqq \sum\left(\begin{array}{c}
m \\
\mu
\end{array}\right) \sum\left(\begin{array}{l}
n \\
\nu
\end{array}\right) \sum_{j_{1}, \cdots, j_{\iota}}\left|D_{x}^{m-\mu} D_{t}^{n-\nu} a_{j_{1}} \ldots j_{s}(0,0)\right| J
$$

where

$$
J=D_{x}^{\mu} D_{t}^{\prime}\left[\left(D_{x}^{q_{1}} D_{t}^{r_{1}} u\right)^{j_{1}} \cdots\left(D_{x}^{q_{t}} D_{t}^{r_{t}} u\right)^{j_{g_{t}}}\right] .
$$

Here $q_{h}=r_{h}=0$, for some $h$, may also be admitted.

For any fixed $i, 1 \leqq i \leqq s$, we introduce

$$
W(x, t)=D_{x}^{q_{i}} D_{t}^{r_{i}} u(x, t) .
$$

Then, for $\sigma<n+p$, we have by the inductive assumption,

$$
\begin{aligned}
\left|D_{x}^{\rho} D_{t}^{\sigma} W(0,0)\right| & \leqq H_{0} H^{\rho+2 \sigma+q_{i}+2 r_{i}-q^{\prime}-2 r^{\prime}-1}\left(\delta \rho+\sigma+\delta q_{i}+r_{i}-\delta q-r-4\right) ! \\
& \leqq H_{0} H^{\rho+2 \sigma-1}(\delta \rho+\sigma-4) !
\end{aligned}
$$

where in the last inequality we made use of the definitions (5.1), (5.2). The inequalities (5.7) show that we can use Lemma 1 . We thus obtain, if $j_{i} \geqq 1$,

$$
\left|D_{x}^{\rho} D_{t}^{\sigma}\left(D_{x}^{q_{i}} D_{t}^{r_{i}} u\right)^{j_{i}}\right| \leqq\left(\frac{K H_{0}}{H}\right)^{j_{i}-1} H_{0} H^{\rho+2 \sigma-1}(\delta \rho+\sigma-4) !
$$


We now proceed by induction on the index $s$ appearing in the definition of $J$, assuming first that all the $j_{i}$ are 1 .

For $s=1$ we have nothing to prove. For $s=2$ we have, using (5.8),

$$
\begin{aligned}
&|J| \leqq \sum_{\alpha}\left(\begin{array}{l}
\mu \\
\alpha
\end{array}\right) \sum_{\beta}\left(\begin{array}{l}
\nu \\
\beta
\end{array}\right)\left|D_{x}^{\alpha} D_{t}^{\beta}\left(D_{x}^{q_{1}} D_{t}^{r_{1}} u\right)^{j_{1}}\right|\left|D_{x}^{\mu-\alpha} D_{t}^{\nu-\beta}\left(D_{x}^{q_{2}} D_{t}^{r_{2}} u\right)^{j_{2}}\right| \\
& \leqq\left(\frac{K H_{0}}{H}\right)^{j_{1}+j_{2}-2} H_{0} H^{\mu+2 \nu-1} \sum_{\alpha} \sum_{\beta}\left(\begin{array}{l}
\mu \\
\alpha
\end{array}\right)\left(\begin{array}{l}
\nu \\
\beta
\end{array}\right)(\delta \alpha+\beta-4) ! \\
& \cdot(\delta \mu-\delta \alpha+\nu-\beta-4) ! \\
& \leqq C\left(\frac{K H_{0}}{H}\right)^{j_{1}+j_{2}-2} H_{0} H^{\mu+2 \nu-1}(\delta \mu+\nu-4) !,
\end{aligned}
$$

where the last inequality follows by the calculation (of the $I$ ) in $\S 3$. For $s=3$ we write

$$
\begin{aligned}
J= & \sum\left(\begin{array}{l}
\mu \\
\alpha
\end{array}\right) \sum\left(\begin{array}{l}
\nu \\
\beta
\end{array}\right)\left[D_{x}^{\alpha} D_{t}^{\beta}\left(\left(D_{x}^{q_{1}} D_{t}^{r_{1}} u\right)^{j_{1}}\left(D_{x}^{q_{2}} D_{t}^{r_{2}} u\right)^{j_{2}}\right)\right] \\
& \times\left[D_{x}^{\mu-\alpha} D_{t}^{\gamma-\beta}\left(D_{x}^{q_{2}} D_{t}^{r_{3}} u\right)^{j_{3}}\right]
\end{aligned}
$$

then proceed as in the case $s=2$, making use of (5.8), (5.9). We obtain

$$
|J| \leqq C\left(\frac{K H_{0}}{H}\right)^{j_{1}+j_{2}+j_{3}-3} H_{0} H^{\mu+2 \nu-1}(\delta \mu+\nu-4) !
$$

It is now clear that $J$ can be estimated in the same manner for any given $s$. If not all the $j_{i}$ are $\geqq 1$, then some of them are $=0$, so that the corresponding factors $\left(D_{x} q_{i} D_{t} r_{i}\right)^{j_{i}}$ are replaced by 1 . The previous procedure of establishing an estimate of $J$ is clearly unchanged. Substituting the estimate on $J$ into (5.6) we obtain after simple calculation (as in $\$ 4,(4.5),(4.6)$ ),

$$
\left|D_{x}^{m} D_{t}^{n+p} u(0,0)\right| \leqq H_{0} H^{m+2 n}(\delta m+n-4) !
$$

(5.10) implies (5.3) with $k=n+p$ if

$$
\begin{aligned}
m+2 n & \leqq m+2 n+2 p-q^{\prime}-2 r^{\prime}-1, \\
\delta m+n-4 & \leqq \delta m+n+p-\delta q-r-4 .
\end{aligned}
$$

In view of (5.1), (5.2) these inequalities are equivalent to

$$
2\left(p-r_{k}\right) \geqq q_{k}+1, \quad \delta q_{j} \leqq p-r_{j},
$$

and these last inequalities are clearly satisfied. The proof of Theorem 2 is thus completed. 


\section{Part II. The Case of a system}

We shall now prove a generalization to systems of the $\mathrm{C}-\mathrm{K}$ theorem. The method is an extension of the method of Part I. The classical proof of the $\mathrm{C}-\mathrm{K}$ theorem for general systems makes a reduction to the case of first order systems. In our method, such a reduction is not needed:

We are concerned with the system

$$
\begin{array}{r}
D_{t}^{p_{i}} u_{i}=f_{i}\left(x, t, u_{1}, \cdots, u_{p}, \cdots D_{z}^{q_{i j j}} D_{i}^{r_{i c j}} u_{c} \cdots\right), \quad(1 \leqq i \leqq \rho), \\
D_{t}^{k} u_{i}(x, 0)=0, \quad\left(0 \leqq k \leqq p_{i}-1,1 \leqq i \leqq \rho\right),
\end{array}
$$

where $x$ varies in a bounded region $B$. The indices $p_{i}, q_{i \sigma j}, r_{i o j}$ must satisfy the conditions

$$
p_{i} \geqq q_{i \sigma j}+r_{i \sigma j}, \quad p_{\sigma}>r_{i \sigma j}, \quad \text { for all } i, \sigma, j .
$$

ThEOREM 3. Let the functions $f_{i}(x, t, v)\left(v=\left(v_{1}, \cdots, v_{s_{i}}\right)\right.$ with appropriate $\left.s_{i}\right)$ belong to class $(\delta, 1,1)$ in the region defined by $x \in B,|t| \leqq t_{0}, \sum v_{i}^{2} \leqq v_{0}^{2}$ for some $t_{0}>0, v_{0}>0$, where $1 \leqq \delta \leqq \min _{i, o, j}\left(p_{i}-r_{i \sigma j}\right) / q_{i \sigma j}$. Then there exists one and only one solution $u_{i}(x, t)(1 \leqq i \leqq \rho)$ of (1), (2) which is analytic in $t$ near $t=0$. Furthermore, the $u_{i}(x, t)$ are of class $(\delta, 1)$ for $x \in B,|t| \leqq t_{1}$ where $t_{1}>0$ is sufficiently small.

As in Part I, Theorem 3 can easily be generalized to the case of nonhomogeneous initial conditions:

$$
D_{t}^{k} u_{i}(x, 0)=\phi_{i k}(x), \quad\left(0 \leqq k \leqq p_{i}-1,1 \leqq i \leqq \rho\right),
$$

provided the $\phi_{i k}(x)$ belong to class $\delta$. Indeed, all we need to do is to change the unknown functions $u_{i}$ by introducing

$$
v_{i}(x, t)=u_{i}(x, t)-\sum_{k=1}^{p_{i-1}} \frac{t^{k}}{k !} \phi_{i k}(x),
$$

and then apply Theorem 3 to the new system in the $v_{i}$.

Proof. Let $\left(q_{i}, r_{i}\right)$ be the a pair of $\left(q_{i \sigma j}, r_{i \sigma j}\right)$ which satisfies

$$
\delta q_{i}+r_{i}=\max _{\bullet, j}\left(\delta q_{i \sigma j}+r_{i \sigma j}\right)
$$

and let $\left(\bar{q}_{i}, \overline{\boldsymbol{r}}_{i}\right)$ be a pair of $\left(q_{i \sigma j}, r_{i \sigma_{j}}\right)$ which satisfies

$$
\bar{q}_{i}+2 \bar{r}_{i}=\max _{\sigma, j}\left(q_{i o j}+2 r_{i \sigma j}\right) .
$$

Following the method of Part I, we shall prove by induction on $n$ that for $1 \leqq i \leqq \rho$,

(8) $\left|D_{x}^{m} D_{t}^{n+p_{i}} u_{i}(x, 0)\right| \leqq H_{0} H^{m+2 n+2 p_{i}-\bar{a}_{i-2_{i}^{-}-1}^{-1}}\left(\delta m+n+p_{i}-\delta q_{i}-r_{i}-4\right) !$ 
for all $m$ such that $m+2 n+2 p_{i} \geqq \bar{q}_{i}+2 \bar{r}_{i}+1$. In view of (2), (8) is satisfied for $n \leqq 0$. Hence it is enough to prove that if (8) holds with $n+p_{i}$ replaced by $k_{i}$, for any $k_{i}<n+p_{i}$, and if $n \geqq 0$, then (8) is satisfied.

We take $H_{0}$ such that

$$
\left|D_{x}^{m} D_{t}^{k+p_{i}} u(x, 0)\right| \leqq H_{0} \text { whenever } m+2 k+2 p_{i} \leqq \bar{q}_{i}+2 \overline{\boldsymbol{r}}_{i}+1 .
$$

From now on $H_{0}$ is fixed. $H$ will be determined in the inductive passage from $k_{i}<n+p_{i}$ to $k_{i}=n+p_{i}$. The case $n<p_{i}-r_{i \sigma j}$ can be settled quite easily. It will be enough to consider the case $n \geqq p_{i}-r_{i \sigma j}$ for all $i, \sigma, j$.

Similarly to the proof of Theorem 2, we write

$$
f_{i}=\sum a_{j_{1}}^{i} \ldots j_{s}(x, t) \cdots\left(D_{x}^{q_{i \sigma j}} D_{t}^{r_{i \sigma j}} u_{\sigma}\right)^{j_{\tau}} \cdots,
$$

where, for simplicity, we may take $s_{i}=s$ (in the formulation of Theorem 3). In order to estimate

$$
D_{x}^{m} D_{t}^{n+p_{i}} u_{i}=D_{x}^{m} D_{t}^{n} f_{i}
$$

we first introduce, for each $(i, \sigma, j)$,

$$
W=D_{x}^{q_{i \sigma j}} D_{t}^{r_{i \sigma j}} u_{\sigma}
$$

By the inductive assumptions and the definitions (6), (7), we have for any $m \geqq 0$,

$$
\begin{aligned}
\left|D_{x}^{m} D_{t}^{k} W\right| & =\left|D_{x}^{m+q_{i \sigma j}} D_{t}^{k+r_{i \sigma j}} u_{\sigma}\right| \\
& \leqq H_{0} H^{m+q_{i \sigma j}+2 k+2 r_{i \sigma j}-\overline{-}_{i}-2 \bar{r}_{i}-1}\left(\delta m+\delta q_{i \sigma j}+k+r_{i \sigma j}-\delta q_{i}-r_{i}-4\right) ! \\
& \leqq H_{0} H^{m+2 k-1}(\delta m+k-4) ! .
\end{aligned}
$$

Hence, we can apply Lemma 1 in order to estimate

$$
D_{x}^{\mu} D_{t}^{\prime}\left(D_{x}^{q_{i \sigma j}} D_{t}^{r_{i \sigma j}} u_{\sigma}\right)^{j_{\tau}} \text { provided } \mu \leqq m, \nu \leqq n .
$$

By calculation very similar to that involving (5.6)-(5.10) of Part I, we then obtain

$$
\left|D_{x}^{m} D_{t}^{n+p_{i}} u_{i}\right| \leqq H_{0} H^{m+2 n}(\delta m+n-4) !, \quad(1 \leqq i \leqq \rho) .
$$

In order to complete the proof of (8) we have to verify that

$$
\begin{aligned}
m+2 n & \leqq m+2 n+2 p_{i}-\bar{q}_{i}-2 \bar{r}_{i}-1, \\
\delta m+n-4 & \leqq \delta m+n+p_{i}-\delta q_{i}-r_{i}-4 .
\end{aligned}
$$

These inequalities reduce to

$$
2\left(p_{i}-\bar{r}_{i}\right) \geqq \bar{q}_{i}+1, \quad \delta q_{i} \leqq p_{i}-r_{i} .
$$


Using the definitions (6), (7), these inequalities reduce to

$$
2\left(p_{i}-r_{i \sigma j}\right) \geqq q_{i \sigma j}+1, \quad \delta q_{i r k} \leqq p_{i}-r_{i r k}
$$

for appropriate $\sigma, j$ and $\tau, k$. Now the first inequality of (14) follows from(3). The second inequality of (14) follows from the assumption on $\delta$ made in Theorem 3. The proof is thus completed.

\section{Part III. A problem of Goursat}

We shall generalize the results. of Parts I, II by considering the case of several $t$ variables. We shall consider the so called Goursat problem in its most general form:

$$
\begin{aligned}
& \text { (1) } D_{t_{1}}^{p_{1}} D_{t_{2}}^{p_{2}} \cdots D_{t_{\sigma}}^{p_{\sigma}} u(x, t)=f\left(x, t, u, \cdots D_{x}^{q_{i}} D_{t_{1}}^{r_{1 i}} \cdots D_{t_{\sigma}}^{r_{o_{i}}} u, \cdots\right) \\
& \text { (2) }\left.D_{t_{j}}^{k} u(x, t)\right|_{t_{j}=0}=\phi_{j k}(x, t), \quad\left(0 \leqq k \leqq p_{j}-1,1 \leqq j \leqq \sigma\right),
\end{aligned}
$$

where the following assumptions must be made:

$$
\begin{gathered}
p_{\lambda}>r_{\lambda i} \text { and } \sum_{j=1}^{\sigma} p_{j} \geqq q_{i}+\sum_{j=1}^{\sigma} r_{j i}, \quad \text { for all } \lambda, i . \\
\left.D_{t_{i} \phi_{j k}}^{m}\right|_{t_{i}=0}=\left.D_{t_{j} \phi_{i m}}^{k}\right|_{t_{j}=0}, \quad \text { for all } i \neq j ; i, j=1, \cdots, \sigma .
\end{gathered}
$$

We have used the notation: $t=\left(t_{1}, \cdots, t_{\sigma}\right)$. For simplicity we shall consider only the homogeneous conditions:

$$
\left.D_{t_{j}}^{k} u(x, t)\right|_{t_{j=0}}=0, \quad\left(0 \leqq k \leqq p_{j}-1,1 \leqq j \leqq \sigma\right) .
$$

The general case then follows using an appropriate transformation. For $\sigma=2$ it is:

$$
v(x, t)=u(x, t)-\sum_{j=1}^{2} \sum_{k=2}^{p_{j}-1} \frac{t_{j}^{k}}{k !} \phi_{j k}(x, t)+\sum_{k=0}^{p_{1}-1} \sum_{l=0}^{p_{2}-1} \frac{i_{1}^{k}}{k !} \frac{t_{2}^{l}}{l !} D_{t_{1}}^{k} \phi_{2 l}(x, 0) .
$$

THEOREM 4. Let $f(x, t, v)\left(v=\left(v_{1}, \cdots, v_{s}\right)\right)$ be of class $(\delta, 1,1)$ for $x \in B$, $\sum t_{i}^{2}<t_{0}^{2}, \quad \sum v_{i}^{2}<v_{0}^{2}$ where $B$ is a bounded region, $t_{0}>0, v_{0}>0$, and let $1 \leqq \delta$ $\leqq \min _{i}\left(p_{1}+\cdots+p_{\sigma}-r_{1 i}-\cdots-r_{o i}\right) / q_{i}$. Then there exists one and only one solution $u(x, t)$ of (1), (5) which is analytic in $t$ near $t=0$. Furthermore, the solution is of class $(\delta, 1)$ for $x \in B, \sum t_{i}^{2}<\tilde{t}$ for some $i>0$ sufficiently small.

This theorem can also be extended to systems of equations.

Proof. For the sake of clarity we shall give the proof only in a special typical case. The extension of the proof to the general case will be omitted since it is similar to what we did in Part I, §5. The case we consider is that of $\sigma=2$, and the equation is

$$
D_{t_{1}}^{p_{1}} D_{t_{2}}^{p_{2}}=f\left(x, t, D_{x}^{q} D_{t_{1}}^{r_{1}} D_{t_{2}}^{r_{2}} u\right)
$$


We proceed by induction to prove that

(8) $\left|D_{x}^{m} D_{t_{1}}^{k_{1}} D_{t_{2}}^{k_{2}} u(x, 0,0)\right|$

$$
\leqq H_{0} H^{m+2 k_{1}+2 k_{2}-q-2 r_{1}-2 r_{2}-1}\left(\delta m+k_{1}+k_{2}-\delta q-r_{1}-r_{2}-6\right) ! .
$$

Once (8) is established for all $m, k_{1}, k_{2}$, then the proof of the theorem can be completed by an argument quite similar to that of Part I, $\$ 2$.

The proof of (8) can be carried first by induction on $k_{1}$ with $k_{2}$ being fixed and then by induction on $k_{2}$. It will be enough to describe the first induction, since the second one is proved in the same way. We thus assume that (8) has been proved for all $k_{1}<n+p_{1}$ and for $k_{2}$ and all the natural numbers smaller than $k_{2}$, and proceed to prove it for $k_{1}=n+p_{1}$ and for $k_{2}$ and all the natural numbers smaller than $k_{2}$. It will be enough to establish the proof only for $k_{1}=n+p_{1}$ with $k_{2}$. Thus we have to prove that

$$
\begin{aligned}
& \left|D_{x}^{m} D_{t_{1}}^{n+p_{1}} D_{t_{2}}^{k_{2}} u(x, 0,0)\right| \\
& \quad \leqq H_{0} H^{m+2 n+2 p_{1}+2 k_{2}-q-2 r_{1}-2 r_{2}-1}\left(\delta m+n+p_{1}+k_{2}-\delta q-r_{1}-r_{2}-6\right) ! .
\end{aligned}
$$

It is enough to take $x=0$.

Using the equation (7) and expanding $f(x, t, v)$ into power series in $v$, we find that

$$
D_{x}^{m} D_{t_{1}}^{n+p_{1}} D_{t_{2}}^{k_{2}} u(0,0)=\left[D_{x}^{m} D_{t_{1}}^{n} D_{t_{2}}^{k_{2}-p_{2}} \sum_{i} a_{i}(x, t)\left[D_{x}^{q} D_{t_{1}}^{r_{1}} D_{t_{2}}^{r_{2}} u(x, t)\right]^{i}\right]_{x=0, t=0} .
$$

We now introduce the function

$$
W(x, t)=D_{x}^{q} D_{t_{1}}^{r_{1}} D_{t_{2}}^{r_{2}} u(x, t)
$$

and find, by the inductive assumption, that

$$
\left|D_{x}^{\mu} D_{t_{1}}^{\nu} D_{t_{2}}^{\lambda} W\right| \leqq H_{0} H^{\mu+2 \nu+2 \lambda-1}(\delta \mu+\nu+\lambda-6) !, \quad\left(\mu \leqq m, \nu \leqq n, \lambda \leqq k_{2}-p_{2}\right) \text {. }
$$

Hence we can apply a slightly modified version of Lemma 1 and obtain, after some calculation similar to that in Part I, §4,

$$
\left|D_{x}^{m} D_{t_{1}}^{n+p_{1}} D_{t_{2}}^{k_{2}} u\right| \leqq H_{0} H^{m+2 n+2 k_{2}-2 p_{2}}\left(\delta m+n+k_{2}-p_{2}-6\right) ! .
$$

(9) would follow from (10), if

$$
\begin{aligned}
m+2 n+2 k_{2}-2 p_{2} & \leqq m+2 n+2 p_{1}+2 k_{2}-q-2 r_{1}-2 r_{2}-1, \\
\delta m+n+k_{2}-p_{2}-6 & \leqq \delta m+n+p_{1}+k_{2}-\delta q-r_{1}-r_{2}-6 .
\end{aligned}
$$

These inequalities reduce to

$$
2\left(p_{1}+p_{2}-r_{1}-r_{2}\right) \geqq q+1, \quad \delta q \leqq\left(p_{1}+p_{2}-r_{1}-r_{2}\right) .
$$


The last two inequalities however follow from (3) and the assumption of the theorem concering $\delta$.

\section{PART IV. Mixed PROBlems}

In recent papers $[8 ; 9]$ Duff considered mixed problems for linear systems by first studying the analytic case and then, by approximation, generalizing it to nonanalytic systems of hyperbolic type. The analytic system can be written, after some reduction, in the form

$$
\begin{array}{rlrl}
D_{t} v_{r} & =\sum \alpha_{r s} D_{\sigma} v_{s}+L_{r}\left(v_{s}, w_{s}\right)+f_{r}, & (1 \leqq r \leqq R-\mu), \\
D_{\sigma} w_{r} & =L_{r}\left(v_{s}, w_{s}\right)+f_{r}, & (R-\mu+1 \leqq r \leqq R), \\
v_{r} & =\sum c_{r \lambda} w_{\lambda}+g_{r}, & (1 \leqq r \leqq R-\mu) \text { on } t=0, \\
w_{\lambda}=\sum d_{\lambda r} v_{r}+g, & (R-\mu+1 \leqq r \leqq \mu) \text { on } \sigma=0,
\end{array}
$$

where $\alpha_{r s}, c_{r \lambda}, d_{\lambda_{r}}$ are analytic functions in $(x, \sigma, t)$ and the $L_{r}$ are linear functions of $v_{s}, w_{s}, D_{x} v_{s}, D_{x} w_{s}$ with coefficients analytic in $(x, \sigma, t)$. Here and in the following, $x=\left(x_{1}, \cdots, x_{N}\right)$, and $\sigma, t$ are real variables.

The system (1) is solved in [8] by the method of majorants of C-K; the majorant problem however is far more complicated than that constructed for the C-K system. In this part we extend our method and derive existence of analytic solutions for nonlinear systems which contain (1) as a very special case.

For simplicity we first consider a special but typical case, namely,

$$
\begin{aligned}
D_{t} v & =f\left(x, \sigma, t, v, w, D_{x} v, D_{x} w, D_{\sigma} v\right), \\
D_{\sigma} w & =g\left(x, \sigma, t, v, w, D_{x} v, D_{x} w\right), \\
v & =h(x, \sigma, w), \\
w & =k(x, t, v),
\end{aligned}
$$$$
\text { on } t=0 \text {, }
$$$$
\text { on } \sigma=0 \text {. }
$$

We assume that (4), (5) hold for $(x, \sigma, t, v, w)=\left(0,0,0, v^{0}, w^{0}\right)=A$ and that $1+h_{w} k_{v} \neq 0$ at $A$. We first reduce the above system to a new system with $h \equiv k \equiv 0$.

By (4) we have, for $t=0$,

$$
v=h, \quad D_{x} v=h_{x}+h_{w} D_{x} w .
$$

Substituting this into (3) we obtain

$$
D_{\sigma} w=\hat{g}\left(x, \sigma, w, D_{x} w\right), \quad \quad \text { for } t=0,
$$

with appropriate $g$. Under the assumption that $f, g, h, k$ are analytic functions in appropriate domains, we conclude that $\vec{g}$ is analytic and that the solutions

$$
v(x, 0,0)=\phi_{1}(x), \quad w(x, 0,0)=\phi_{2}(x),
$$

of (4), (5) are also analytic. Hence, the equation (6) with the initial condition 
$w=\phi_{2}$ has an analytic solution $w(x, \sigma, 0) \equiv \psi_{1}(x, \sigma)$ near $(x, \sigma)=(0,0)$.

Substituting this solution into (4) we get

$$
v(x, \sigma, 0)=h\left(x, \sigma, \psi_{1}(x, \sigma)\right) \equiv \psi_{2}(x, \sigma) .
$$

We now perform the transformation

$$
\tilde{v}(x, \sigma, t)=v(x, \sigma, t)-\psi_{2}(x, \sigma), \quad \tilde{w}(x, \sigma, t)=w(x, \sigma, t)-k(x, t, v(x, \sigma, t)) .
$$

Clearly, this is a reversible transformation, that is, $v$ and $w$ can be calculated in terms of $\tilde{\tau}, \tilde{w}$. Hence, if we prove existence of an analytic solution $\tilde{\boldsymbol{v}}, \tilde{w}$, then the existence of an analytic solution $v, w$ will follow. It is easily seen that $\tilde{v}, \tilde{w}$ satisfy a system of the form

$$
\begin{aligned}
& D_{t} v=f\left(x, \sigma, t, v, w, D_{x} v, D_{x} w, D_{\sigma} v\right), \\
& D_{\sigma} w=g\left(x, \sigma, t, v, w, D_{x} v, D_{x} w, D_{\sigma} v\right),
\end{aligned}
$$

with $v, w, f, g$ replaced by $\tilde{\sigma}, \tilde{w}, \bar{f}, \tilde{g}$.

We have thus proved that the system (2)-(5) is reducible to a system composed of (7), (8) and

$$
\begin{aligned}
& v(x, \sigma, 0)=0 \\
& w(x, 0, t)=0 .
\end{aligned}
$$

We shall state our theorem for the reduced system (7)-(10).

THEOREM 5. Let $f, g$ be analytic functions in all their variables in a neighborhood of the origin. Then the system (7)-(10) has one and only one analytic solution $v(x, \sigma, t), w(x, \sigma, t)$ in a neighborhood of the origin.

Proof. We wish to prove by induction on $n$ that

$$
\begin{array}{r}
\left|D_{x}^{l} D_{\sigma}^{m} D^{n} u\right|_{\sigma=l=0} \leqq H_{0} H_{1}^{l^{\prime}-3}(1+m+n-4) !,\left(l^{\prime}=\max (l, 3)\right), \\
\text { for } l \geqq 0, m \leqq 3, n \leqq 2, \\
\left|D_{x}^{l} D_{\sigma}^{m} D_{t}^{n} u\right|_{\sigma=t=0} \leqq H_{0} H_{1}^{l-3} H_{2}^{m-3}(l+m+n-4) !, \\
\quad \text { for } l \geqq 0, m \geqq 4, n \leqq 2, \\
\left|D_{x}^{l} D_{\sigma}^{m} D_{t}^{n} u\right|_{\sigma=l=0} \leqq H_{0} H_{1}^{l-3} H_{2}^{m-3} H_{3}^{n-2}(l+m+n-4) !, \\
\quad \text { for } l \geqq 0, m \geqq 0, n \geqq 3,
\end{array}
$$

for $u=v, w$. Here, the functions $D_{x}^{l} D_{\sigma}^{m} D_{\imath}^{n} u$ are determined successively by differentiating the equations (7), (8). Once $\left(11^{\prime \prime}\right)$ and (11) are established, the proof of Theorem 5 is completed by extending the argument of Part I, $\$ 2$.

To derive $\left(11^{\prime}\right)$, we make use of (9), (10) and of Lemma 1 (slightly modified). Since this is quite similar to the derivation of $\left(11^{\prime \prime}\right)$ and (11) below, we omit details. From now on, $H_{0}, H_{1}$ are fixed, and $H_{1} \geqq 1$. The proof 
of $\left(11^{\prime \prime}\right)$ for $n=0, u=v$ is trivial in view of (10). The proof of $\left(11^{\prime \prime}\right)$ for $n=0, u=w$ follows by using (7), (9), (10) and Lemma 1 (slightly modified). To derive it for $n=1, u=v$, we apply $D_{x}^{l} D_{\sigma}^{m} D_{t}$ to (7) and make use of $\left(11^{\prime}\right)$, of $\left(11^{\prime \prime}\right)$ with $n=0$ and of Lemma 1 (slightly modified). Next we apply $D_{x}^{l} D_{\sigma}^{m} D_{t}$ to (8) and estimate $D_{x}^{l} D_{\sigma}^{m+1} D_{t}^{2} w$ by induction on $m$, the expression $D_{x}^{l} D_{t}^{2} w$ being zero, by (10). The case $n=2$ is treated similarly. We shall not describe here a detailed derivation of $\left(11^{\prime \prime}\right)$ since it is similar to the derivation of (11). We now proceed to derive (11) by induction on $n$. We assume it for $n$ and prove it for $n+1$. Note that $H_{1}, H_{2}$ are now fixed, and that $H_{2}$ will be determined in the passage from $n$ to $n+1$ (independently of $n$ ). We may assume, to begin with, that $H_{2}>H_{1}^{3}, H_{3}>H_{1}^{3} H_{2}^{3}$.

We apply $D_{x}^{l} D_{\sigma}^{m} D_{t}^{n}$ to (7) and make use of the inductive assumptions and of Lemma 1 (slightly modified). We obtain after some calculation (provided $H_{3}$ is sufficiently large, independently of $\left.l, m, n\right)$,

$$
\left|D_{x}^{l} D_{\sigma}^{m} D_{t}^{n+1} v\right| \leqq K_{1} H_{0} H_{1}^{l-2} H_{2}^{m-2} H_{3}^{n-2}(l+m+n-3) !
$$

where $K_{i}$ are used to denote constants independent of $H_{1}, H_{2}, H_{3}, l, m, n$. In order to complete the estimation of $v$ for $n+1$, we only have to take $H_{3}>K_{1} H_{1} H_{2}$.

We turn to $w$. By (10), $D_{x}^{l} D_{i}^{k} w=0$ at $\sigma=t=0$, for all $l, k$. We proceed to estimate $D_{x}^{l} D_{\sigma}^{m} D_{t}^{n+1} w($ at $\sigma=t=0)$ by induction on $m$. We assume that

$$
\left|D_{x}^{l} D_{\sigma}^{\mu} D_{t}^{n+1} w\right| \leqq K H_{0} H_{1}^{l-2} H_{2}^{\mu-2} H_{3}^{n-2}(l+\mu+n-3) !
$$

for all $\mu \leqq m$ and proceed to prove it for $\mu=m+1$. Applying $D_{x}^{l} D_{\sigma}^{m} D_{t}^{n+1}$ to (8) and using the inductive assumptions, (12) and Lemma 1 (in a slightly modified form), we obtain after some calculation

$$
\begin{aligned}
\left|D_{x}^{l} D_{\sigma}^{m+1} D_{t}^{n+1} w\right| \leqq & \left(K_{3} K_{1} H_{0} H_{1}^{l-2} H_{2}^{m-1} H_{3}^{n-2}+K_{3} K H_{0} H_{1}^{l-2} H_{2}^{m-2} H_{8}^{n-2}\right. \\
& \left.+K_{4} H_{0} H_{1}^{l-2} H_{2}^{m-1} H_{3}^{n-2}\right)(l+m+n-2) !
\end{aligned}
$$

where $K_{3}, K_{4}$ are independent of $K$. Here we made use of the fact that $H_{1}$, $\mathrm{H}_{2}$ are sufficiently large (right from the beginning) depending only on $g$, and of the inequality $H_{2}>H_{1}$. To complete the proof of (13) by induction on $\mu$, we only have to assume at the beginning of the proof that

$$
K>3 K_{3} K_{1}, \quad H_{2}>3 K_{3}, \quad K>3 K_{4} \text {. }
$$

Having proved (13) for all $\mu$, we take $H_{3}>K H_{1} H_{2}$, and the proof of (11) for $n$ replaced by $n+1$ and $u=w$ is completed.

Theorem 5 can be immediately extended to the case where $v=\left(v_{1}, \cdots, v_{\mu}\right)$, $w=\left(w_{1}, \cdots, w_{\nu}\right)$ and the proof is essentially the same. This extension can be carried out also for the system (2)-(5). We shall now consider systems of higher order equations of the form 


$$
\begin{array}{rc}
D_{t_{1} v_{i}}^{p_{i}}=f_{i}\left(x, t, \cdots, D_{x}^{q_{i j \tau}} D_{t_{1}}^{r_{i j \tau}} D_{t_{2}}^{i_{i j \tau}} v_{\tau}, \cdots, D_{x}^{Q_{i j \tau}} D_{t_{1}}^{R_{i j \tau}} D_{t_{2}}^{\mathcal{S}_{i j \tau}} w_{\tau}, \cdots\right), & (i=1, \cdots, \mu), \\
D_{t_{2}}^{\tilde{p}_{i} w_{i}}=g_{i}\left(x, t, \cdots, D_{x}^{\tilde{q}_{i j \tau}} D_{t_{1}}^{\tau_{i j \tau}} D_{t_{2}}^{\tilde{s}_{i j \tau}} v_{\tau}, \cdots, D_{x}^{\tilde{Q}_{i j \tau}} D_{t_{1}}^{\tilde{R}_{i j \tau}} D_{t_{2}}^{\boldsymbol{S}_{i j \tau}} w_{\tau}, \cdots\right) & (i=1, \cdots, \nu), \\
D_{t_{1} v_{i}}^{k}=0, & \left(0 \leqq k \leqq p_{i}-1 ; 1 \leqq i \leqq \mu\right) \text { on } t_{1}=0, \\
D_{t_{2} w_{i}}^{k}=0, & \left(0 \leqq k \leqq \tilde{p}_{i}-1 ; 1 \leqq i \leqq \nu\right) \text { on } t_{2}=0 .
\end{array}
$$

Here $t=\left(t_{1}, t_{2}\right)$. We assume that

$$
\begin{gathered}
p_{i} \geqq q_{i j \tau}+r_{i j \tau}+s_{i j \tau}, \quad p_{i} \geqq Q_{i j \tau}+R_{i j \tau}+S_{i j \tau}, \\
\tilde{p}_{i} \geqq \tilde{q}_{i j \tau}+\tilde{r}_{i j \tau}+\tilde{s}_{i j \tau}, \quad \tilde{p}_{i} \geqq \tilde{Q}_{i j \tau}+\tilde{R}_{i j \tau}+\tilde{S}_{i j \tau}, \\
p_{\tau}>r_{i j \tau}, \quad p_{\tau}>R_{i j \tau}, \quad p_{\tau}>\tilde{r}_{i j \tau}, \quad p_{\tau}>\tilde{R}_{i j \tau}, \quad \tilde{p}_{\tau}>\tilde{S}_{i j \tau} .
\end{gathered}
$$

We then can prove:

If the $f_{i}, g_{j}$ are analytic in all their variables in a neighborhood of the origin, then the system (15)-(18) has one and only one solution $v_{i}(x, t), w_{j}(x, t)$ analytic in $(x, t)$ in a neighborhood of the origin.

The proof is based on the proof of Theorem 5, and makes use of some technical devices used in the proofs of Theorems 2,3 .

We remark that the last result can be extended to the case of nonhomogeneous initial values and also to equations with certain degree to nonanalyticity in $x$. Finally, it can be extended to the case $t=\left(t_{1}, \cdots, t_{M}\right)$.

\section{REFERENCES}

1. A. Cauchy, Mémoire sur un théorème fondamental, dans le calcul intégral, C. R. Acad. Sci. Paris vol. 39 (1842) pp. 1020-1026.

2. - Memoire sur l'emploi du calcul des limites dans l'intégration des équations aux dérivées partielles, C. R. Acad. Sci. Paris vol. 40 (1842) pp. 44-59.

3. - Mémoire sur l'application du calcul des limites a l'intégration d'un système d'équations aux dérivées partielles, C. R. Acad. Sci. Paris vol. 40 (1842) pp. 85-101.

4. - Mémoire sur les systèmes d'équations aux dérivées partielles d'ordres quelconques, et sur leur réduction dè des systèmes d'équations linéares $d u$ premier ordre, C. R. Acad. Sci. Paris vol. 40 (1842) pp. 131-138.

5. - Note sur divers theorèmes relatifs aux calcul des limites, C. R. Acad. Sci. Paris vol. 40 (1842) pp. 138-139.

6. - Mémoire sur les intégrales des systèmes d'équations différentielles et aux dérivées partielles, et sur le devéloppement de ces intégrales en séries ordonnés suivant les puissances ascendantes d'un paramètre que renferment les équations proposées, C. R. Acad. Sci. Paris vol. 40 (1842) pp. 141-146.

7. G. Darboux, Sur l'existence de l'intégrale dans les équations aux dérivées partielles d'ordre quelconque, C. R. Acad. Sci. Paris vol. 80 (1875) pp. 317-318.

8. G. F. D. Duff, Mixed problems for linear systems of the first order equations, Canad. J. Math. vol. 10 (1958) pp. 127-160.

9. - Mixed problems for hyperbolic equations of general order, Canad. J. Math. vol. 11 (1959) pp. 195-221. 
10. V. R. Friedlender and G. S. Salehov, On the question of the inverse of the CauchyKowalewski problem, Uspehi Mat. Nauk SSSR vol. 7 (1952) pp. 169-192.

11. V. R. Friedlender, On the Cauchy-Kowalewski problem for some partial differential equations, Uspehi Mat. Nauk SSSR vol. 12 (1957) pp. 385-388.

12. - On the analyticity of solutions of the Cauchy problem for some nonlinear partial differential equations, Mat. Sb. (N.S.) vol. 47 (1959) pp. 17-44.

13. A. Friedman, On the regularity of the solutions of nonlinear elliptic and parabolic systems of partial differential equations, J. Math. Mech. vol. 7 (1958) pp. 43-59.

14. M. Gevrey, Sur la nature analytique des solutions des equations aux deriveses partielles, Ann. Ecole Norm. Sup. vol. 35 (1918) pp. 129-190.

15. E. Goursat, Sur l'existence des fonctions integrales d'un système d'equations aux dérivées partielles, Bull. Soc. Math. France vol. 26 (1898) pp. 129-134.

16. - Cours d'analyse mathematique, vol. II, 1905.

17. E. Holmgren, Sur l'equation de la propagation de la chaleur, Arkiv für Math. Astr. Physik vol. 4 (1908) pp. 1-2, 3-4.

18. S. Kowalewski, Zur Theorie der partiellen Differentialgleichungen, J. Reine Angew. Math. vol. 80 (1875) pp. 1-32.

19. P. D. Lax, Nonlinear hyperbolic equations, Comm. Pure Appl. Math. vol. 4 (1953) pp. 231-258.

20. Le Roux, Sur les intégrales analytiques de l'equation $\partial^{2} u / \partial y^{2}=\partial u / \partial x$, Bull. Sci. Math. France vol. 19 (1895) pp. 127-129.

21. C. Pucci, Nuove ricerche sul problema di Cauchy, Mem. Accad. Sci. Torino. Cl. Sci. Fis. Mat. Nat. (3) vol. 1 (1953) pp. 45-67.

University of Minnesota, Institute of Technology, MinNeAPOLIS, MinNESOTA 